ISSN: 2162-3104 Print/ ISSN: 2166-3750 Online

Volume 8, Issue 2 (2018), pp. 960-976

(C) Journal of International Students

http://jistudents.org/

doi: 10.5281/zenodo. 1250395

\title{
Student Perceptions of Providers' Cultural \\ Competence, Attitudes Towards Providers, and Patient Satisfaction at a University Health Center: International and U.S. Student Differences
}

\author{
Nicole C. Hudak \\ Ohio University, USA \\ Heather J. Carmack \\ The University of Alabama, USA \\ Ethan D. Smith \\ Independent Scholar, USA
}

\begin{abstract}
The present study surveyed international and domestic U.S. students about their perceptions of university health care providers' cultural competence, their own attitudes toward university health center providers, and overall satisfaction with university health center providers. Results demonstrated generally positive attitudes and satisfaction, but more negative perceptions of provider cultural competence were identified especially when students perceived problems with providers' language competence and interest in learning about patients' backgrounds. International students reported more positive attitudes concerning providers' cultural competence than U.S. students. Implications of the findings are discussed, along with limitations of the study and suggestions for future research.
\end{abstract}

Keywords: college health, cultural competency, health communication, international students, patient satisfaction 
The increasing complexity and diversification of the United States and the globalization catalyzed by major innovations in transportation and communication technology bring the importance of cultural competency to the forefront of communication study and practice (Perloff, Bonder, Ray, Ray, \& Siminoff, 2006). To be culturally competent, communicators must now consider a number of complex social variables, such as heritage, culture, ethnicity, religion, socialization, and identity, all within contexts of diversity, demographic change, population, immigration patterns, and poverty (Spector, 2012). In consideration of such realities, cultural competence involves understanding and attending to the total context of those with whom a communicator interacts, combining a constant development and improvement of knowledge, attitudes, and skills (Spector, 2012). The development of this competence is an arduous and timeconsuming process, to be sure, but one which is becoming increasingly essential.

Understanding cultural competence is often no more apparent than at institutions of higher learning where individuals from various fields of experience gather and interact with, live with, and learn from one another (Gurin, Dey, Hurtado, \& Gurin, 2002). However, students do not only have influential interaction with their peers while pursuing their degree; they also interact with university faculty and staff, including university health center (UHC) providers. Cultural competence is especially important in interactions between medical providers and patients. Cultural competence in health care recognizes the perspectives of both providers and patients in order to foster understanding and identify cross-cultural differences (Ahmed \& Bates, 2007, 2010; Ahmed, Bates, \& Romina, 2016). This enables providers and patients to work together to pursue medical options which are culturally-sensitive and can achieve desired health outcomes (Ahmed \& Bates, 2010). Researchers have found that higher quality provider-patient relationships are associated with higher patient satisfaction levels (Campbell et al., 2007). Cultural competency's impact on health outcomes highlights the need for health care providers who serve diverse populations to be especially aware of their patients' cultural backgrounds and their own abilities to communicate across cultural divides.

Scholars have examined students' perceptions of instructor cultural competence (e.g., Brennan \& Cotter, 2008), yet no studies to date have explored student perceptions of UHC providers' cultural competence. Such inquiry is important, as the cultural competency of UHC providers can 
impact the health outcomes of student patients. This is particularly true as U.S. university populations include increasing numbers of international students. The present study attempts to develop a greater understanding of the complex and interrelated variables which influence student patient satisfaction with UHC provider care. Specifically, the researchers examine the intersection of personal and cultural variables and student patient perceptions of UHC providers' cultural competency, student attitudes toward providers, and student patient satisfaction with their UHC providers. The article begins by discussing cultural competency and patient satisfaction before reporting on the results of a quantitative survey examining significant differences in perception among international and domestic U.S. college students.

\section{LITERATURE REVIEW}

Patient satisfaction is considered a hallmark outcome of health care (Williams, 1994) Patient satisfaction is defined by eight dimensions: art of care, technical quality of care, accessibility/convenience, finances, physical environment, availability, continuity of care, and efficacy/outcomes of care (Ware, Davies-Avery, \& Stewart, 1977). The study of patient satisfaction in health care has been primarily focused on large hospital facilities and clinics (Williams, Weinman, \& Dale, 1998), but the limited research on college students and patient satisfaction suggests that they are concerned with some, but not all, of these dimensions. Deshwal and colleagues (2014) observed that dimensions affecting college students' patient satisfaction include staff professionalism, clinic staff reliability, clinic accessibility and basic facilities, tangibles, cleanliness, awareness of the clinic, and how clinic staff deals with emergencies. More than $70 \%$ of the respondents in this study reported that the clinic staff paid good attention to them, more than $60 \%$ reported that the clinic doctor and staff were competent, and $50 \%$ of the respondents deemed the campus clinic reliable (Deshwal et al., 2014). Half the respondents in the Deshwal et al. (2014) study did not approve of clinic facilities, which the authors interpreted as patients feeling that clinic equipment was not up-to-date or visually appealing.

College students are, however, concerned with the relatability of their providers. Students patients who viewed their providers as friendlier rather than hostile were more satisfied with their visits than patients who viewed their providers as more hostile than friendly (Campbell et al., 2007). 
The patient-centered model emphasizes the need for providers to engage in quality interpersonal relationships with patients. Campbell et al. (2007) determined that higher quality provider-patient relationships are associated with higher patient satisfaction levels. Student patients desire providers who are highly friendly, minimally hostile, and more submissive than dominant (Campbell et al, 2007). Student patients also desired to have their preferences taken into account by providers, the ability to be empowered, and feeling competent in managing their own health outcomes (Campbell et al., 2007). The researchers argued that perceived student patient satisfaction was related to age and the medical situation: younger, more educated individuals typically dealing with relatively mundane health issues are more likely to be satisfied with their care (Campbell et al., 2007).

A number of variables have been identified which negatively impact college students' perception of satisfaction with care. A study performed by a student health center at a university in Wisconsin found that wait times are a major component of student patient satisfaction; students with shorter waits perceived the staff as kinder and more compassionate, despite no interventions to influence those factors (Eilers, 2004). Pressures to contain costs due to decreased state funding and limited student service fees can also be a challenge for university health center providers who do not wish to sacrifice quality of care (Anderson, 1995). Studies of UHCs in Texas and North Carolina found that students were more satisfied with the reliability, assurance, and responsiveness of their care then they were with provider empathy and helpfulness of UHC staff (Anderson, 1995; Canel \& Anderson Fletcher, 2001). Those challenges and that pressure to contain costs without sacrificing quality still apply to UHC today. Universities with higher percentages of on-campus residents may have an advantage over commuter schools; on-campus residents may have to use their UHC because they do not have transportation to off-campus health care facilities. However, offcampus health care facilities can lure students living off-campus by providing the non-medical dimensions that affect patient satisfaction and that UHCs may not be able to provide, such as parking or later hours. It is important to note that all of these studies focus on UHC services and their connection to patient satisfaction. Missing from these discussions is an indepth examination of relational variables which may impact patient satisfaction. 


\section{Cultural Competency}

Cultural diversity is a core part of the social and economic make-up of the United States and has implications for the delivery of health care (Perloff et al., 2006; Spector, 2012). Culture is important in health care because it plays a role in creating health-related values, beliefs, and behaviors (Betancourt, 2004). Individual cultural background, which includes a person's heritage and language, impacts how a patient accesses and responds to health care services and how providers conduct their practice (Spector, 2012). Due to the role that culture plays in health care, health care providers have become increasingly aware of the need for cultural competence to meet the needs of their patients (Spector, 2012).

Patient-provider interactions are often instances of cross-cultural communication, highlighting the importance of the influence of cultural competencies across various health domains. In order to address the gap that exists between culture and health care providers, providers may attempt to develop their own cultural competence. Ahmed and Bates (2010) noted that cultural competence in health care is a process that acknowledges both the health care providers' and health care receivers' perspectives while promoting knowledge and recognition of cross-cultural differences in order to adapt to the current health care situation. Cultural competency is a complex process, as it takes time to develop the skills, knowledge, and attitudes necessary to become culturally competent in order to truly hear, understand, and respect the needs and perspectives of a patient (Spector, 2012). Providers need to continually strive to improve their cultural competence skills (Perloff et al., 2006). These skills can be improved in a number of ways, including understanding a person's personal heritage, the heritage of others, health beliefs and practices of culturally diverse others, the culturally diverse health care culture and system, and the traditional health care system (Spector, 2012).

Cultural competence may be conflated with cultural appropriateness and/or cultural sensitivity. Spector (2012) explained the differences between the three definitions. Cultural competence is when a provider delivers care, understanding and adhering to the context of a patient's situation with a complex combination of knowledge, attitudes, and skills. Cultural appropriateness refers to when a provider applies his or her cultural knowledge in order to provide a patient with the best possible health care. Finally, cultural sensitivity is when a provider has a basic knowledge of attitudes toward the health traditions of a diverse cultural group. An 
emphasis on cultural competency assumes that health services can reduce health disparities, that interventions are more accessible, acceptable, and effective when culturally adapted, and that practitioners have the propensity to acquire the knowledge, attitudes, and skills to improve delivery of culturally competent care (Kimayer, 2012). The focus, then, is less on the development of full-fledged empathetic understanding with various cultures and more on knowledge that allows for providers to make improvements to health care systems and/or processes. However, in addition to cultural awareness, knowledge, and skills, other models include personal cultural encounters and a caring desire to want to work with other cultures in order truly help (Campinha-Bacote, 2002).

Training health care professionals in cultural competence can be effective, improving knowledge, attitudes, and skills of the provider, and influencing patient satisfaction (Beach et al., 2005). Sherrill and Mayo (2014) assessed medical and nursing students' cultural competence, communication skills, and overall readiness to treat Latino patients. The researchers found that the students did not have adequate knowledge of Latino populations and even had some discomfort in treating Latino patients. However, they also found that those students who had clinical experiences with Latino patients were more comfortable in treating them, suggesting that cultural competence can be built through continual contact.

The socially constructed nature of cultural competence suggests it is a skill which may be refined in order to produce improved health outcomes and patient satisfaction. The increase in the diversity of student patients requires UHC providers to be able to competently adapt to patients, while still providing them compassionate care. Thus, this study attempted to investigate student patients' perceptions of their providers' cultural competence and patient satisfaction and how these students' perceptions, attitudes, and satisfaction differ from those of their domestic counterparts. The following hypotheses guided this study:

- H1: There will be significant differences between international and U.S. students' perceptions of university health center providers' cultural competence.

- H2: There will be significant differences between international and U.S. students' attitudes towards university health center providers. 
- H3: There will be significant differences between international and U.S. students' satisfaction with university health center provider care.

\section{RESEARCH METHOD}

\section{Participants}

A total of 267 students participated in the study. Participants were all undergraduate students attending a large Mid-Atlantic public university. One hundred seventy-five females (65.5\%) and 91 males (34.1\%) completed the survey, with one student reporting "Other" $(0.4 \%)$. Most participants were $18(\mathrm{n}=135,50.6 \%)$ or $19(\mathrm{n}=116,43.4 \%)$ years old; a limited number of participants were 20 years old and older $(\mathrm{n}=16,6 \%)$. Participants included first-year $(\mathrm{n}=253,94.8 \%)$, second-year $(\mathrm{n}=7,2.6 \%)$, third-year $(\mathrm{n}=4,1.5 \%)$, and fourth-year $(\mathrm{n}=3,1.1 \%)$ students, of whom all but one were full-time students $(\mathrm{n}=266,99.6 \%)$.

Respondents were predominantly white/Caucasian $(n=227,85 \%)$, with a number of students identifying as Hispanic/Latino $(\mathrm{n}=13,4.9 \%)$, black/African-American $(\mathrm{n}=12,4.5 \%)$, Asian $(\mathrm{n}=10,3.7 \%)$, Pacific Islander $(\mathrm{n}=2,0.7 \%)$, and "Other" $(\mathrm{n}=1.1 \%) .92 .1 \%$ of students reported being from the United States $(n=246)$, while 21 reported international origins $(7.9 \%)$. International countries represented included China $(\mathrm{n}=3$, $1.1 \%)$, Ghana $(n=2,0.7 \%)$, India $(n=2,0.7 \%)$, Jamaica $(n=2,0.7 \%)$, and the United Kingdom $(\mathrm{n}=2,0.7 \%)$, with ten other nations around the world each represented by a single student $(0.4 \%)$, including Bolivia, Bulgaria, Columbia, the Dominican Republic, Italy, the Netherlands, Panama, Peru, Sweden, and Vietnam. 94.8\% of students reported being straight/heterosexual $(\mathrm{n}=253)$, while gay and lesbian students $(\mathrm{n}=3$, $1.1 \%)$, bisexual students $(\mathrm{n}=5,1.9 \%)$, and students who selected the "Other" option ( $\mathrm{n}=6,2.2 \%)$ were less represented.

Participants must have visited the university health center at least once during the school year. Students most often reported visiting the UHC one to two times $(\mathrm{n}=209,78.3 \%)$, although many students visited three to five times $(n=46,17.2 \%)$, six to eight times $(n=9,3.4 \%)$, and nine or more times $(\mathrm{n}=3,1.2 \%)$. Students mostly reported being seen by a physician or doctor $(\mathrm{n}=130,48.7 \%)$, although some also met with a nurse practitioner ( $\mathrm{n}$ $=51,19.1 \%)$, physician assistant $(\mathrm{n}=14,5.2 \%)$, nurse $(\mathrm{n}=12,4.5 \%)$, or some other provider $(\mathrm{n}=4,1.5 \%)$. However, $21 \%$ of students reported 
being unsure of what type of provider provided their primary treatment $(\mathrm{n}=$ 56). Reasons for visiting the health center were largely described in terms of severity as "not that bad" $(\mathrm{n}=99,37.1 \%)$ or "bad" $(\mathrm{n}=95,35.6 \%)$, although some students also reported their reason for visitation as "very severe" $(n=6,2.2 \%)$, "severe" $(n=24,9 \%)$, or "not bad" $(n=30,11.2 \%)$. Thirteen students reported their reason as "a routine visit" $(4.9 \%)$.

\section{Data Collection and Instrumentation}

Participant recruitment and data collection began after the researchers received approval from their university's Institutional Review Board. Students were recruited in two ways: through an online research databank and through emails requesting participation from the university's Office of International Programs. Participation in the study involved the completion of an online survey, which involved demographic information and three main areas of measurement: student patient perceptions of health care provider cultural competence, student patient attitudes toward the provider, and patient satisfaction. The survey was administered using Qualtrics, an online websurveying software program.

\section{Patients' Perceptions of Physicians' Cultural Competence}

To measure perceptions of cultural competence, respondents completed the Patients' Perception of Physicians' Cultural Competence scale (PPPCC; Ahmed \& Bates, 2010), a 20-item, five subscale instrument. The PPPCC uses a 5-point Likert type scale with lower scores indicating greater perceived cultural competence of the provider. The overall scale was reliable, with a Cronbach alpha of 0.91 .

The physician global cultural competence (PGCC) is comprised of three areas: macro-cultural issues, proxemics/chronemics, and language. The PGCC macro-cultural issues subscale is a 5-item, 5-point Likert type scale, found to be reliable $(\alpha=0.92)$. Sample statements include "My provider wants to know about my religious practices related to health issues" and "My provider asks me who makes important decisions in my family." Lower scores indicate greater perceived physician cultural competence of macro-cultural issues.

The PGCC proxemics/chronemics subscale was a reliable $(\alpha=0.82)$ 3-item, 5-point Likert type scale which included items such as "My provider asks me if I would feel discomfort if the provider touches me during the physical exam" and "My provider wants to know if time is a concern for me 
with regard to medical treatment." Lower scores indicate greater perceived provider proxemic/chronemic cultural competence.

The PGCC language subscale included three items measured on a 5point Likert type scale, included items such as "My provider wants to know about my language skills" and "My provider considers using the help of available translators". Lower scores indicate greater perceived physician linguistic cultural competence. The subscale was reliable $(\alpha=0.91)$.

The physician's patient-centered cultural competence (PPCCC) subscale is a 4-item, 5-point Likert type scale, including statements such as "My provider tries to understand my feelings" and "My provider wants to know my viewpoint on treatment goals." Lower scores indicate greater perceived PPCCC. The PPCCC subscale was observed to be reliable, with a Cronbach alpha score of 0.89 .

Finally, the patient satisfaction subscale is a 5-item, 5-point Likert type scale where participants evaluated different elements related to their most recent health center visit from "Excellent" to "Poor". The subscale also included statements such as "Explanation of what was done for you" and "The personal manner of the provider you saw." Lower scores indicate greater patient satisfaction. The patient satisfaction subscale was reliable $(\alpha$ $=0.95$ ).

\section{Attitudes Toward Provider}

To measure attitudes, participants completed an adapted form of the Attitudes Toward Provider scale (ATP; Hajek, Villagran, \& WittenbergLyles, 2007), which consisted of six items measured on a 5-point Likert type scale. The ATP focused on perceived respectfulness of the provider and perceived quality of provider explanations (two questions), the extent to which they perceived the provider to be similar or different from other providers (one question), and their inclinations to comply with provider recommendations (three questions). Higher scores indicate more positive attitudes toward providers. The overall scale was reliable, with a Cronbach alpha of 0.76 . The compliance inclination subscale was reliable $(\alpha=0.80)$.

\section{RESULTS}

Data were analyzed with the Statistical Package for the Social Sciences (SPSS 21). Independent samples t-tests and analysis of variance (ANOVA) tests were performed to answer the study's three hypotheses. See Table 1 for means and standard deviations for the measures. Overall, students reported 
the perceived UHC providers' cultural competence to be slightly worsethan-average, with scale and subscale averages indicating overall poor perceptions of cultural competence. This worse-than-average result is a result of students specifically reporting unfavorable perceptions of providers' language and macro-cultural issues. Students were more favorable of providers' patient-centeredness. They also reported more favorable responses for patient satisfaction. Participants reported overall positive attitudes toward providers and positive inclinations to follow provider recommendations.

Table 1. Means and Standard Deviations of Scales and Subscales

\begin{tabular}{|c|c|c|c|c|}
\hline & $N$ & $M$ & $S D$ & Cronbach's $\alpha$ \\
\hline Overall PPPCC & 267 & 3.04 & 0.61 & 0.91 \\
\hline $\begin{array}{l}\text { PPPCC- Patient } \\
\text { Satisfaction }\end{array}$ & 267 & 2.36 & 1.01 & 0.95 \\
\hline $\begin{array}{l}\text { PPPCC- Patient } \\
\text { Centeredness }\end{array}$ & 267 & 2.66 & .087 & .089 \\
\hline PPPCC- Language & 267 & 3.70 & .086 & .091 \\
\hline $\begin{array}{l}\text { PPPCC- Macrocultural } \\
\text { issues }\end{array}$ & 267 & 3.69 & .79 & .092 \\
\hline $\begin{array}{l}\text { PPPCC- } \\
\text { Proxemics/chronemics }\end{array}$ & 267 & 2.95 & .91 & .082 \\
\hline Overall ATP & 267 & 3.56 & .062 & 0.76 \\
\hline $\begin{array}{l}\text { ATP- Inclination to } \\
\text { follow orders }\end{array}$ & 267 & 3.20 & .079 & 0.80 \\
\hline
\end{tabular}

PPPCCC = Patients' perceptions of providers' cultural competence $\mathrm{ATP}=$ Attitude toward provider

The first hypothesis stated that there would be differences between international and U.S. students' perceptions of university health center providers' cultural competence. The independent samples t-tests supported this hypothesis. The t-test for total patient perceptions of provider cultural competence was found be significant, $\mathrm{t}(265)=-5.02, \mathrm{p}=.000$. U.S. students $(\mathrm{M}=3.76, \mathrm{SD}=.76)$ perceived providers to be significantly less competent with macro-cultural issues than international students $(\mathrm{M}=2.90, \mathrm{SD}=.74)$. The t-test for patient perception of provider proxemics-chronemics competence was also found be significant, $\mathrm{t}(265)=-1.99, \mathrm{p}=.048$. U.S. students $(\mathrm{M}=2.98, \mathrm{SD}=.91)$ perceived providers to be significantly less 
competent with proxemics-chronemic issues than international students $(\mathrm{M}$ $=2.57, \mathrm{SD}=.78$ ). The t-test for patient perception of provider language competence was found be significant, $\mathrm{t}(265)=-5.46, \mathrm{p}=.000$. U.S. students $(\mathrm{M}=3.78, \mathrm{SD}=.83)$ perceived providers to be significantly less competent with language issues than international students $(\mathrm{M}=2.76, \mathrm{SD}=.74)$.

The second hypothesis posited that there would be differences between international and U.S. students' attitudes towards UHC providers. Only one significant difference existed between international students and U.S. students. A t-test for inclination to comply with provider recommendations was found be significant, $\mathrm{t}(265)=2.06, \mathrm{p}=.040$. U.S. students $(\mathrm{M}=3.17, \mathrm{SD}=.79)$ were significantly less inclined to comply with provider recommendations than international students $(\mathrm{M}=3.54, \mathrm{SD}=$ .74). No other significant differences between groups were found.

Finally, hypothesis three stated that international students and U.S. students would report different levels of patient satisfaction. This hypothesis was not supported, $\mathrm{t}(265)=.192, \mathrm{p}=.88$. International students $(\mathrm{M}=2.33$, $\mathrm{SD}=1.02)$ and U.S. students $(\mathrm{M}=2.41, \mathrm{SD}=1.03)$ both report overall satisfaction with UHC provider care.

\section{DISCUSSION AND CONCLUSIONS}

Increasing globalization has highlighted the growing need for cultural competency in health care interactions (Spector, 2012; Perloff et al., 2006). This is particularly essential for the health care provided on college campuses (Gurin et al., 2002). This study offers the first foray into student perceptions of UHC providers' cultural competence, focusing on differences between international and native U.S. students. Across the board, students believed UHC providers has below average cultural competence, even though they were overall satisfied with their care.

Overall, international students perceived UHC providers as significantly more competent than U.S. students, both in total cultural competency, as well as across the global cultural competence areas of macro-cultural issues, language, and proxemics-chronemics. This difference may be because UHC providers know these students are from outside the United States and alter their communication approach in order to be more culturally competent. The UHC providers may skip over questions about a student's nationality, cultural background, religious practices related to health issues, language preferences, discomfort with being touched during 
the physical exam, and viewpoints on illness and treatment goals with U.S. students because of provider assumptions that they have similar beliefs, backgrounds, and cultural preferences; in short, providers may perceive those questions as obvious and unnecessary when it comes to U.S. students.

Another explanation may be associated with the cultural background of UHC providers. It is possible that providers at this UHC are from other countries, in which case, international students might view those providers as relatable and feel more accepted, while U.S. students might experience ambivalence, or potential discomfort. Previous research suggests that generally, U.S. patients are not as comfortable with and biased against foreign-born providers (Hoff, 2009; Louis et al., 2010), so it is possible this is could impact U.S. students' perceptions.

International students also reported higher perceived provider cultural competence related to macro-cultural issues. The macro-scale included questions that related to religious practices, nationality, cultural background, and individual decision making. It is possible that these topics were considered more important for international students than U.S. students. Providers do ask about race and nationality on intake forms or during medical exams, but they do not typically ask about patients' religious practices, cultural background, or decision-making processes, although research suggests patients would be open to being asked about their religion affiliation or cultural background (Ehman, Ott, Short, Ciampa, \& HansenFlaschen, 1999; Hasnain-Wynia \& Baker, 2006). Asking these questions is important because culture, religion, and nationality can impact how patients communicate with providers and what information they disclose during exams (Spector, 2012).

International students were also significantly more likely to comply with the university health center providers' recommendations for care. This could be due to differences with past medical providers, or due to cultural differences concerning expected respect and reverence given to medical providers. U.S. students may have more of a preconceived hierarchy of medical professionals ingrained in their understanding of health care (Carmack, 2014), meaning providers at the UHC could be perceived as less respected than those at a hospital or private practice. It also could be a combination of U.S. students being non-complaint and believing that providers are not always correct. Campbell et al. (2007) found that perceived provider competence among student patients in managing their own health outcomes was correlated with students' decreased willingness to 
cede control to medical providers. Students from the United States also might be more likely to "shop around" for the provider, diagnosis, or treatment that they, or their parents, prefer. International students may want different qualities in their providers, they may not feel that they can fire their medical provider, or not know that there are other options available besides the UHC (Carmack, Bedi, \& Heiss, 2016).

\section{Limitations and Future Directions}

The present study had several limitations. Participants were overwhelmingly female, straight/heterosexual, white/Caucasian, first-year students who were between 18 and 19 years of age. Also, a majority of respondents reported being from the United States. Although similar demographics have been used previously to study patients' perceptions of cultural competence (Ahmed \& Bates, 2007, 2010; Ahmed et al., 2016), cultural competence results could be different with more diverse samples. The international student sample was also small. Although the authors strategically recruited with the Office of International Programs and emails to international students, the sample was still not as robust as the native U.S. student sample. Second, the study relied on self-reported data from student participants. It does not include information about UHC providers' cultural background or level of expertise. This information would certainly add another layer to the complex nature of cultural competence and patient satisfaction.

Third, there are inconsistencies with the PPPCC subscales. While the patient satisfaction subscale is supposed to be a part of the total PPPCC scale, satisfaction would seem to be more of an outcome of provider cultural competence rather than a part of it. Additionally, it became somewhat unclear what larger theoretical concept the subscale proxemics-chronemics is attempting to measure; the proxemics item appears to be measuring haptics and the chronemics items actually measure time, which are two distinct concepts. This uncertainty complicates researchers' ability to understand and theorize about the subscale, which serves as an obstacle to developing a more complete picture of the nature of cultural competence in health.

The results of the present study also allow for directions for future research. Future research must more closely examine differences in perceptions, attitudes, and competencies by culture. This study combined all international students into one group and did not take into consideration the 
unique health care experiences specific to different regions of the world. Such work would begin to reveal the complexities of differences between U.S. and international students, but also identify differences amongst international students. For example, research could explore if simply being an international student is indicative of stronger PPPCC, or if there are differences across different cultures. This could help UHC with large numbers of international student patients from specific countries or cultures identify important cultural care practices. Future research should also compare student and provider perceptions of cultural competence related to patient satisfaction. Hailey, Pargeon, and Crawford (2000) found inconsistencies between student patient and UHC providers' ratings of patient satisfaction. Although they theorized as to why these discrepancies existed, more research is needed to improve student patient satisfaction. Likewise, it is important to talk with UHC providers about their perceptions of cultural competence and identify ways to increase their competency.

Patient perception of providers' cultural communication competence is an essential element of ideal health care provision, with the potential to impact health outcomes. The present study found generally positive attitudes and communication inclinations toward university health center providers, but poorer perceptions of overall cultural competence. Analysis demonstrated that U.S. students typically have poorer perceptions of the cultural competency of university health center providers than international students. That international students see UHC providers as more culturally competent than native U.S. students is a somewhat surprising result. This finding serves as a call for more research on UHC provider cultural competence as well as the need for competency training.

\section{REFERENCES}

Ahmed, R., \& Bates, B. R. (2007). Gender differences in patients' perceptions of physicians' cultural competence in health care interactions. Women's Health \& Urban Life, 6(2), 58-80. Retrieved from http://www.utsc.utoronto.ca/ womenshealth/womenshealth/Home.html

Ahmed, R., \& Bates, B. R. (2010). Assessing the relationship between patients' ethnocentric views and patients' perceptions of physicians' cultural competence in health care interactions. Intercultural Communication Studies, 19(2), 111-127. Retrieved from http://web.uri.edu/iaics/iaicsjournal/ 
Ahmed, R., Bates, B. R., \& Romina, S. M. (2016). Assessing the influence of patients' perceptions of providers' cultural competence on patient satisfaction in an Appalachian Ohio context. Howard Journal of Communications, $27(4)$, 403-421. http://dx.doi.org/10.1080/10646175.2016.1211569

Anderson, E. A. (1995). Measuring service quality at a university health clinic. International Journal of Health Care Quality Assurance, 8(2), 32-37. doi: http://dx.doi.org/10.1108/09526869510081866

Beach, M. C., Price, E. G., Gary, T. L., Robinson, K. A., Gozu, A., Palacio, A., ...Cooper, L. A. (2005). Cultural competency: A systematic review of health care provider educational interventions. Medical Care, 43(4), 356373. Retrieved from http://journals.lww.com/lwwmedicalcare/pages/default.aspx

Betancourt, J. R. (2004). Becoming a physician: Cultural competence? Marginal or mainstream movement? New England Journal of Medicine, 351, 953-955. Retrieved from http://www.nejm.org/

Brennan, A.M.W., \& Cotter, V.T. (2008). Student perceptions of cultural competence in the curriculum. Journal of Professional Nursing, 24(3), 155-160. http://dx.doi.org/10.1016/j.profnurs.2008.01.003

Campbell, T. A., Auerbach, S. M., \& Kiesler, D. J. (2007). Relationship of interpersonal behaviors and health-related control appraisals to patient satisfaction and compliance in a student health center. Journal of American College Health, 55(6), 333-340. doi:

10.3200/JACH.55.6.333-340

Campinha-Bacote, J. (2002). The process of cultural competence in the delivery of healthcare services: A model of care. Journal of Transcultural Nursing, 13, 181-184. doi: $10.1177 / 10459602013003003$

Canel, C., \& Anderson Fletcher, E. A. (2001). An analysis of service quality at a student health center. International Journal of Health Care Quality Assurance, 14(6), 260-267. https://doi.org/10.1108/09526860110404220

Carmack, H. J. (2014). Ethics in provider-patient interaction. In T. Thompson (Ed.), Encyclopedia of health communication (pp.436-437). Thousand Oaks, CA: Sage.

Carmack, H. J., Bedi, S., \& Heiss, S. N. (2016). International students, university health centers, and memorable messages about health. Journal of International Students, 6(1), 52-72. Retrieved from https://jistudents.org/

Ehman, J. W., Ott, B. B., Short, T. H., Ciampa, R. C., \& Hansen-Flaschen, J. (1999). Do patients want physicians to inquire about their spiritual or religious beliefs if they become gravely ill? Archives of Internal Medicine, 159(15), 1803-1806. doi:10.1001/archinte.159.15.1803.

Eilers, G. M. (2004). Improving patient satisfaction with waiting time. Journal of American College Health, 53(1), 41-48. doi: 10.3200/JACH.53.1.41-48 
Deshwal, P., Ranjan, V., \& Mittal, G. (2014). College clinic service quality and patient satisfaction. International Journal of Health Care Quality Assurance, 27(6), 519-530. http://dx.doi.org/10.1108/IJHCQA-06-20130070

Gurin, P., Dey, E.L., Hurtado, S., \& Gurin, G. (2002). Diversity and higher education: Theory and impact on educational outcomes. Harvard Educational Review, 72(3), 330-366. Retrieved from http://hepg.org/herhome/home

Hailey, B. J., Pargeon, K., \& Crawford, V. (2000). Can healthcare providers at a university health clinic predict patient satisfaction? Journal of American College Health, 49(3), 111-117. http://dx.doi.org/10.1080/07448480009596292

Hajek, C., Villagran, M., \& Wittenberg-Lyles, E. (2007). The relationships among perceived physician accommodation, perceived outgroup typicality, and patient inclinations toward compliance. Communication Research Reports, 24(4), 293-302. doi: 10.1080/08824090701624189

Hasnain-Wynia, R., \& Baker, D. W. (2006). Obtaining data on patient race, ethnicity, and primary language in health care organizations: Current challenges and proposed solutions. Health Services Research, 41(4, pt 1), 1501-1518. doi: 10.1111/j.1475-6773.2006.00552.x

Hoff, T. (2009). Practice under pressure: Primary care physicians and their medicine in twenty-first century. Piscataway, NJ: Rutgers University Press

Kimayer, L. J. (2012). Rethinking cultural competence. Transcultural Psychiatry, 49(2), 149-164. doi: 10.1177/1363461512444673

Louis, W. R., Lalonde, R. N., \& Esses, V. M. (2010). Bias against foreign-born or foreign-trained doctors: Experimental evidence. Medical Education, 44(12), 1241-1247. doi: 10.1111/j.1365-2923.2010.03769.x

Perloff, R.M., Bonder, B., Ray, G. B., Ray, E.B., \& Siminoff, L.A. (2006). Doctorpatient communication, cultural competence, and minority health. American Behavioral Scientist, 49(6), 835-852. doi: $10.1177 / 0002764205283804$

Sherrill, W. W., \& Mayo, R. M. (2014). Medical and nursing student communication skills: Preparing to treat Latino patients. Journal of Communication in Healthcare, 7(2), 128-136. doi: 10.1179/1753807614Y.0000000052

Spector, R. E. (2012). Cultural diversity in health and illness (8th ed.). Upper Saddle River, NJ: Pearson.

Ware, J. E., Davies-Avery, A., \& Stewart, A. L. (1977). The measurement and meaning of patient satisfaction. Health \& Medical Care Services Review, 1(1), 1-3. Retrieved from https://www.ncbi.nlm.nih.gov/labs/journals/health-med-care-serv-rev/ 
Williams, B. (1994). Patient satisfaction: A valid concept? Social Science \& Medicine, 38(4), 509-516. doi:10.1016/0277-9536(94)90247-X

Williams, S., Weinman, J., \& Dale, J. (1998). Doctor-patient communication and patient satisfaction: A review. Family Practice, 15(5), 480-492. doi:10.1093/fampra/15.5.480

NICOLE C. HUDAK, MA, is a doctoral student in the School of Communication Studies, Ohio University. Her major research explores the cultural understanding and influences in health contexts, with a special emphasis in LGBTQ health. Email: nh017915@ohio.edu.

HEATHER J. CARMACK, PhD, is an associate professor in the Department of Communication Studies, The University of Alabama. Her research focuses on communication in health care organizations, communication and patient safety, and cultural issues in health. Email: hjcarmack@ua.edu.

ETHAN D. SMITH, MA, is an independent researcher. Email: ethan.d.smith01@gmail.com.

Manuscript submitted: May 12, 2017

Revised submitted: July 21, 2017 Accepted for publication: October 21, 2017 